Reviews in Mathematical Physics

Vol. 16, No. 5 (2004) 675-677

(C) World Scientific Publishing Company

\title{
ERRATUM
}

\section{A UNIFIED APPROACH TO RESOLVENT EXPANSIONS AT THRESHOLDS}

[Rev. Math. Phys. Vol. 13, No. 6 (2001) 717-754]

Arne Jensen and Gheorghe Nenciu

This note contains an erratum to, and a few remarks on, the paper in the title [1]. The erratum is that the statement of Corollary 2.2 in [1] is incomplete. For the conclusion to be true, one needs that $A_{0} S=0$, which is not the case when $A_{0}$ has a nilpotent part. The correct form is

Proposition 1. Let $F \subset \mathbf{C}$ have zero as an accumulation point. Let $A(z), z \in F$, be a family of bounded operators of the form

$$
A(z)=A_{0}+z A_{1}(z),
$$

with $A_{1}$ uniformly bounded as $z \rightarrow 0$. Suppose 0 is an isolated point of the spectrum of $A_{0}$, and let $S$ be the corresponding Riesz projection. If

$$
A_{0} S=0 \text {, }
$$

then for sufficiently small $z$, the operator $B(z): S \mathcal{H} \rightarrow S \mathcal{H}$ defined by

$$
B(z)=\frac{1}{z}\left(S-S(A(z)+S)^{-1} S\right)=\sum_{j=0}^{\infty}(-z)^{j} S\left[A_{1}(z)\left(A_{0}+S\right)^{-1}\right]^{j+1} S,
$$

is uniformly bounded as $z \rightarrow 0$. The operator $A(z)$ has a bounded inverse in $\mathcal{H}$, if and only if $B(z)$ has a bounded inverse in $S \mathcal{H}$, and in this case

$$
A(z)^{-1}=(A(z)+S)^{-1}+\frac{1}{z}(A(z)+S)^{-1} S B(z)^{-1} S(A(z)+S)^{-1} .
$$

The rest of the paper is not affected, since everywhere $A_{0}$ is self-adjoint, so that (2) holds true.

We remark that (2) might be true, even if $A_{0}$ is not self-adjoint. As we shall prove below, this is the case when one considers the asymptotic expansion of the perturbed 
resolvent around an embedded non-threshold eigenvalue, $\lambda_{0}$, of the unperturbed (self-adjoint) Hamiltonian, as in [2].

More precisely (see [2] for details), if the unperturbed resolvent is

$$
R_{0}(z)=\frac{P_{0}}{\lambda_{0}-z}+\tilde{R}_{0}(z)
$$

and $V=|V|^{1 / 2} U|V|^{1 / 2}$ is a bounded self-adjoint perturbation (here we choose $U$ to be unitary by defining it to be 1 on $\operatorname{Ker} V$, such that $U^{2}=I$ ), then $A_{0}$ turns out to be

$$
A_{0}=U+\lim _{\eta \searrow 0}|V|^{1 / 2} \tilde{R}_{0}\left(\lambda_{0}+i \eta\right)|V|^{1 / 2} .
$$

It is assumed that the limit exists and is compact, and then either $A_{0}$ has a bounded inverse, or 0 is an isolated part of the spectrum of $A_{0}$. In the latter case, one can define the corresponding Riesz projection, $S$.

The next proposition shows that under these conditions, (2) holds true.

\section{Proposition 2.}

$$
S A_{0}=S A_{0} S=0
$$

Proof. The first equality follows from the fact that $S$ is a Riesz projection. The key observation is that from (6)

$$
\operatorname{Im} A_{0} \geq 0 .
$$

Consider now, as an operator in $S \mathcal{H}$,

$$
A_{1}=S A_{0} S .
$$

On one hand $S \mathcal{H}$ is finite dimensional, and on the other hand for all $\Psi \in S \mathcal{H}$

$$
\operatorname{Im}\left\langle\Psi, A_{1} \Psi\right\rangle=\operatorname{Im}\left\langle\Psi, S A_{0} S \Psi\right\rangle=\operatorname{Im}\left\langle\Psi, A_{0} S \Psi\right\rangle=\operatorname{Im}\left\langle\Psi, A_{0} \Psi\right\rangle \geq 0,
$$

i.e.

$$
\operatorname{Im} A_{1} \geq 0 \text {. }
$$

Since $A_{1}$ is nilpotent,

$$
\operatorname{Tr} A_{1}=\operatorname{Tr} \operatorname{Re} A_{1}+i \operatorname{Tr} \operatorname{Im} A_{1}=0,
$$

which together with (10) implies that

$$
\operatorname{Im} A_{1}=0 .
$$

As a consequence, $A_{1}$ is self-adjoint, and since $\sigma\left(A_{1}\right)=\{0\}$, it follows that $A_{1}=0$.

The last remark is that one can generalize Corollary 2.2, and then the whole procedure in [1], to non-self-adjoint $A_{0}$, under the additional assumption that $A_{0}$ is a Fredholm operator with index zero [3] (i.e. $\left.\operatorname{dim} \operatorname{Ker} A_{0}=\operatorname{dim}\left(\operatorname{Ran} A_{0}\right)^{\perp}\right)$. Then 
if $A_{0}=W\left|A_{0}\right|$ is the polar decomposition of $A_{0}$, one can extend (in a non-unique way) $W$ to a unitary operator $U$ (just take $\left\{f_{j}\right\}$ and $\left\{g_{j}\right\}$ orthonormal bases in $\operatorname{Ker} A_{0}$ and $\left(\operatorname{Ran} A_{0}\right)^{\perp}$ respectively, and define $\left.U f_{j}=g_{j}\right)$. Then write

$$
A(z)=U\left(\left|A_{0}\right|+z U^{-1} A_{1}(z)\right)
$$

and apply Corollary 2.2 to $\left|A_{0}\right|+z U^{-1} A_{1}(z)$.

There is a price to pay in this case: due to the non-unicity of $U$, the obtained expansions are not "canonical". Of course, the coefficients of the expansion do not depend on $U$, but various identities have to be used in order to see that.

\section{References}

[1] A. Jensen and G. Nenciu, A unified approach to resolvent expansions at threshold, Rev. Math. Phys. 13 (2001) 717-754.

[2] A. Jensen, On a unified approach to resolvent expansions for Schrödinger operators, in Spectral and Scattering Theory and Related Topics (RIMS, Kyoto, Japan); Sūrikaisekikenkyūsho Kōkyūroku 1208 (2001) 91-103.

[3] T. Kato, Perturbation Theory for Linear Operators (Springer-Verlag, Berlin, 1966). 